\title{
Community health worker knowledge and management of pre-eclampsia in rural Karnataka State, India
}

Umesh Ramadurg ${ }^{1}$, Marianne Vidler ${ }^{2^{*}}$, Umesh Charanthimath $^{3}$, Geetanjali Katageri $^{4}$, Mrutyunjaya Bellad $^{3}$, Ashalata Mallapur ${ }^{4}$, Shivaprasad Goudar ${ }^{3}$, Shashidhar Bannale ${ }^{5}$, Chandrashekhar Karadiguddi $^{3}$, Diane Sawchuck ${ }^{6}$, Rahat Qureshi ${ }^{7}$, Peter von Dadelszen ${ }^{8}$, Richard Derman ${ }^{9}$ and the Community Level Interventions for Pre-eclampsia (CLIP) India Feasibility Working Group

\begin{abstract}
Background: In India, the hypertensive disorders of pregnancy and postpartum haemorrhage are responsible for nearly $40 \%$ of all maternal deaths. Most of these deaths occur in primary health settings which frequently lack essential equipment and medication, are understaffed, and have limited or no access to specialist care. Community health care workers are regarded as essential providers of basic maternity care; and the quality of care they provide is dependent on the level of knowledge and skills they possess. However, there is limited research regarding their ability to manage pregnancy complications. This study aims to describe the current state of knowledge regarding pre-eclampsia and eclampsia among community health care workers (auxiliary nurse midwives, accredited social health activists, staff nurses) in northern Karnataka, India. Furthermore, this study describes the treatment approaches used by various cadres of community health workers for these conditions. The findings of this study can help plan focussed training sessions to build upon their strengths and to address the identified gaps.

Methods: Data were collected as part of a larger study aimed at assessing the feasibility of community-based treatment for pre-eclampsia. Eight focus group discussions were conducted in 2012-2013 in northern Karnataka State: four with staff nurses and auxiliary nurse midwives and four with accredited social health activists. In addition, twelve auxiliary nurse midwives and staff nurses completed questionnaires to explore their competence and self-efficacy in managing pre-eclampsia. Qualitative data were audio-recorded, transcribed verbatim and translated for thematic analysis using NVivo 10.

Results: Community health workers described their understanding of the origins of hypertension and seizures in pregnancy. Psychological explanations of hypertension were most commonly reported: stress, tension, and fear. The most common explanation for eclampsia was not receiving a tetanus vaccination. Despite some common misperceptions regarding aetiology, these community health workers demonstrated a good grasp of the potential consequences of hypertension in pregnancy. According to auxiliary nurse midwives and staff nurses, if hypertension was detected they encouraged rest, decreased salt intake, iron supplementation and tetanus vaccination. In addition, some staff nurses administered antihypertensives, $\mathrm{MgSO}_{4}$, or other anticonvulsants. All auxiliary nurse midwives had some awareness of $\mathrm{MgSO}_{4}$, but none had administered it themselves.

(Continued on next page)
\end{abstract}

\footnotetext{
* Correspondence: marianne.vidler@cw.bc.ca

${ }^{2}$ Department of Obstetrics and Gynaecology, and the Child and Family

Research Unit, University of British Columbia, Vancouver V5Z 4H4, Canada

Full list of author information is available at the end of the article
} 
(Continued from previous page)

Conclusions: This study showed that knowledge regarding the aetiology of pre-eclampsia was limited. Nevertheless, their basic knowledge and skills could be strengthened to more effectively manage the hypertensive disorders of pregnancy in their communities.

Trial registration: NCT01911494

Keywords: Blood pressure, Community health workers, Community health services, Developing countries, Eclampsia, Hypertension, India, Magnesium sulfate, Maternal health, Maternal mortality, Nurse midwives, Pre-eclampsia, Pregnancy, Proteinuria, Public health, Referral and consultation, Rural health

\section{Plain English summary}

In India, pregnancy complications related to hypertension or are responsible for nearly $40 \%$ of all maternal deaths. Most of these deaths occur in primary health facilities which frequently lack essential equipment and medication, are understaffed, and have limited access to specialist care. Community health workers are essential providers of basic maternity care in these settings. The quality of care they provide is dependent on the level of knowledge and skills they possess; however, there is limited evidence regarding their knowledge and confidence in managing pregnancy complications. This study aims to describe the current state of knowledge regarding pre-eclampsia (a complication of pregnancy characterized by high blood pressure, protein in the urine, and/or symptoms) and eclampsia (seizures in pregnancy) among community health workers (auxiliary nurse midwives, accredited social health activists, and staff nurses) in northern Karnataka State, India.

Eight focus group discussions were conducted in 2012-2013: four with staff nurses and auxiliary nurse midwives and four with accredited social health activists. In addition, twelve auxiliary nurse midwives and staff nurses completed questionnaires to explore their skills and confidence in providing obstetric care.

Community health workers provided psychological explanations for the origin of hypertension in pregnancy, such as stress, tension, and fear. Despite some common misperceptions, community health workers demonstrated a good grasp of the potential outcomes related to pregnancy complications. According to auxiliary nurse midwives and staff nurses, if hypertension was detected, they encouraged rest, decreased salt intake, iron supplementation and tetanus vaccination.

This study showed that community health workers had limited knowledge of pre-eclampsia, and their basic knowledge and skills could be strengthened to more effectively manage the hypertensive disorders of pregnancy in their communities.

\section{Background}

India contributes significantly to the global burden of maternal mortality, as it is responsible for $17 \%$ of all maternal deaths [1]. The current maternal mortality ratio (MMR) of India is 167 per 100,000 livebirths and 144 in Karnataka State (SRS 2010-12) [2]. The hypertensive disorders of pregnancy (HDP) are a leading causes of maternal death $[3,4]$, accounting for $10-15 \%$ of all direct maternal deaths globally [3-6]. Most of these deaths occur in low- and middle-income countries (LMIC) [6]. In India, postpartum haemorrhage and the HDPs contribute to nearly $40 \%$ of all maternal deaths [7]. The HDP are challenging because of the silent nature of the disease. Apart from being an important cause of maternal mortality, the HDPs are associated with serious maternal and neonatal morbidities.

Most maternal deaths and morbidities occur in primary health settings which lack essential resources, are understaffed, and have limited or no access to specialist care [5]. In the Indian public health system, primary health centres (PHC) serve as the first point of care. Each PHC is staffed by one doctor and three to five staff nurses, and each sub-centre is staffed by one auxiliary nurse midwife (ANM) [8, 9]. ANMs provide health services including screening, management, and referral for pregnancy and newborn complications. Since 2005, the National Rural Health Mission (NRHM) has introduced innovative strategies to accelerate progress towards improving health outcomes. These strategies include mobilization efforts by frontline workers, namely the accredited social health activists (ASHA), and numerous of initiatives to increase institutional deliveries [10]. In spite of these efforts, Karnataka State has not achieved the expected level of reduction in maternal mortality.

The community health care workers serve as the point of entry into the health system and therefore can play an important role in improving pregnancy outcomes. The proximity of these health workers to their communities is advantageous as it increases familiarity and trust. Ensuring adequate knowledge of pregnancy complications by these health workers is essential. There is limited research available regarding the knowledge and skills among community health care workers (ANM, ASHA, staff nurse) to manage pregnancy complications in India. The aim of this study was to assess the knowledge of community health workers regarding pre-eclampsia and 
eclampsia and how they act upon presentation of these conditions in their communities.

\section{Methods}

This study was conducted in two rural districts (Belgaum and Bagalkot) of northern Karnataka State, India (Fig. 1). Social and health indicators of Karnataka are lagging compared with other South Indian states. Health care infrastructure is often inadequate, particularly in rural areas, where there are insufficient health care providers to cover the large and dispersed population.

There are various cadres of community health workers in India, including ASHAs, ANMs and staff nurses. ASHAs act as community health activists, to counsel women on birth preparedness, the importance of safe delivery, breastfeeding, immunisation, contraception, pregnancy related ailments, sexually transmitted infections and newborn care [11, 12]. ANMs serve a larger population and their primary role is to provide basic maternal and child health care [10]. Staff nurses are trained health workers based at PHCs, they provide preventive and curative services, and are trained as skilled birth attendants [9] (Table 1). Data for this study were collected as part of a larger study aimed at assessing the feasibility of community-based identification and treatment of preeclampsia using community health care workers [13].

\section{Focus group discussions}

Focus group discussions were utilized to elucidate indepth information and to encourage group dialogue. Local clinicians and researchers with no known association with respondents were trained to facilitate these discussions. Eight focus group discussions, four with staff nurses and ANMs and four with ASHAs were conducted at local primary health centres between January and March 2013. Data saturation was felt to be met at this time and no more focus groups were required. The discussions guides were in local language, Kannada.

All discussions were transcribed verbatim in Kannada and translated to English for analysis using NVivo 10 software. Transcripts were coded by one author (MV),

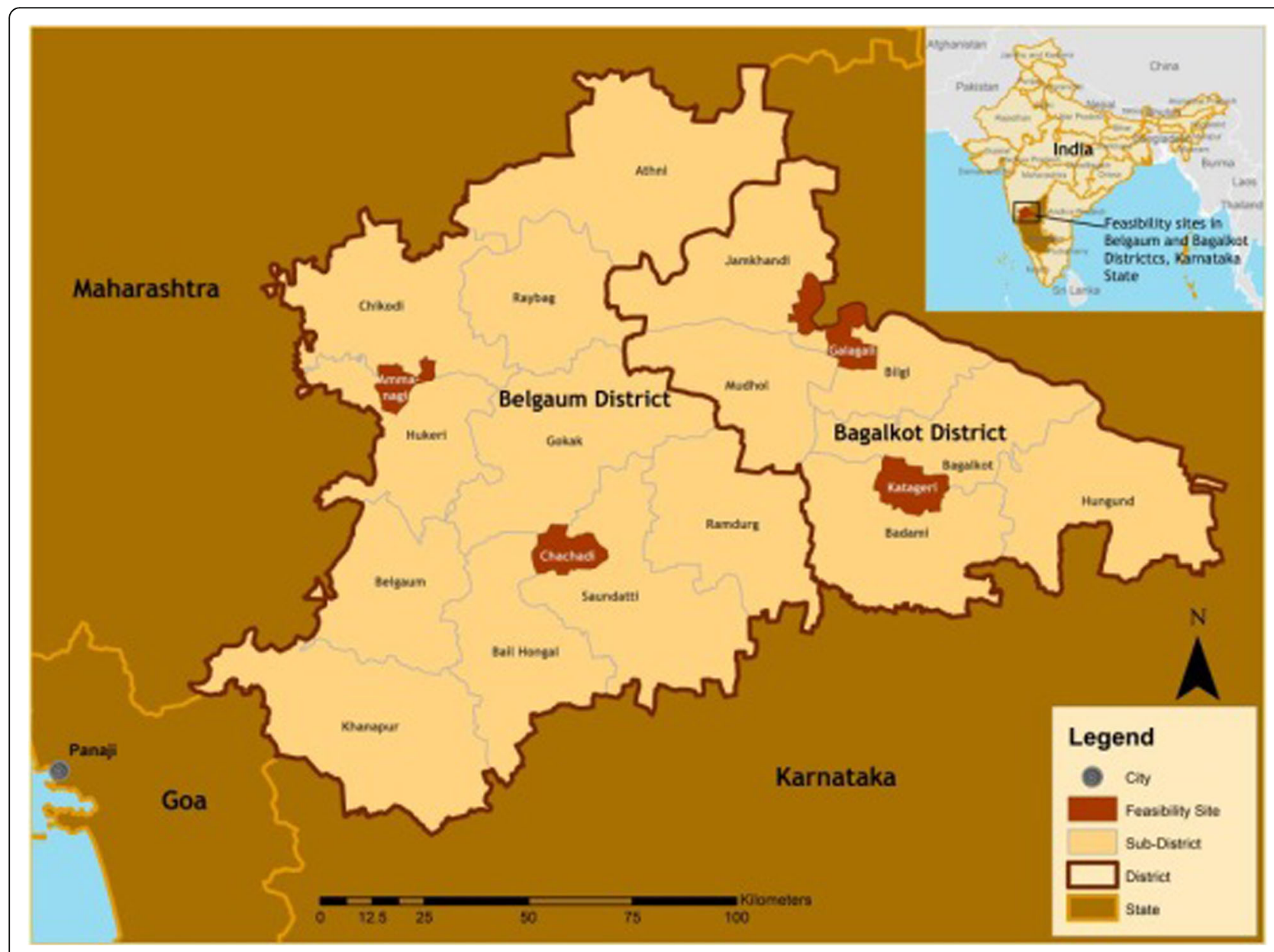

Fig. 1 Study area, Belgaum and Bagalkot, Karnataka State, India 
Table 1 Community health worker characteristics

\begin{tabular}{llll}
\hline & Staff nurse & Auxiliary nurse midwife & Accredited social health activist \\
\hline Minimum education attainment & $\begin{array}{l}\text { General nursing and midwifery training } \\
\text { Bachelor of Science in nursing } \\
\text { Government salary }\end{array}$ & $10^{\text {th }}$ level & $8^{\text {th }}$ level \\
Source of payment & Guxiliary Nurse Midwife training & $\begin{array}{l}\text { Incentives provided through } \\
\text { national health programmes }\end{array}$ \\
Length of training & $24-36$ months & 18 months & 21 days \\
Workplace & Primary health centre & Sub-centre & Village and home \\
Population served & $20,000-30,000$ & $5000-6000$ & 1000
\end{tabular}

after which all transcripts and themes were crosschecked by the local research team (UR, UC, GK, MB, $\mathrm{AM}, \mathrm{KC}$ ) to resolve any misinterpretation. Using deductive reasoning, the results were grouped into predetermined categories related to pre-eclampsia knowledge and skills. During analysis, inductive reasoning was used to incorporate new and unexpected ideas. This produced a comprehensive analysis structure to reflect the richness and variety of responses.

\section{Questionnaires}

Questionnaires were completed by eight ANMs and four staff nurses. Some ANMs were unable to be reached as they were on leave or otherwise occupied. The questionnaire was split into two sections: one with 16 closed-ended Likert scale questions, the other with 11 open-ended questions. The purpose of this assessment was to understand the level of competence and knowledge related to preeclampsia amongst these ANMs and staff nurses.

This study was approved by ethics review committees at the University of British Columbia, Vancouver Canada (H12-00132) and KLE University, Belgaum India (MDC/ IECHSR/2011-12). Written informed consent was obtained for all participants.

\section{Results}

\section{Participants}

A total of 101 community health care workers participated in this study, of which 48 were ANMs or staff nurses and the remaining 53 were ASHAs. The mean age of ANM/staff nurse was $36.5 \pm 4.8$ years and their years of experience ranged from 2 to 27 years. The mean age of ASHAs was $32.5 \pm 0.7$ years and they tended to have 5 years of experience (Table 2).

\section{Danger signs of hypertension and convulsions in pregnancy}

Staff nurses, ANMs and ASHAs explained that to identify pre-eclampsia, blood pressure must be measured; this is the only method of identification as symptoms cannot be used reliably to diagnose. This response showed an understanding that pre-eclampsia can develop without outward symptoms. In addition to hypertension, ANMs claimed that dizziness, swelling, visual disturbances, sweating and restlessness, were danger signs associated with preeclampsia. When probed regarding the signs of eclampsia, the responses included jerky movements, shaking of hands and legs, frothing from the mouth and rolling of the eyes. Both the groups provided similar findings.

\section{Causes of hypertension and convulsions in pregnancy}

There were several explanations for the hypertensive disorders of pregnancy provided by all the three groups of community health workers. Psychological explanations of hypertension were most common: mental stress, tension, and fear. Tensions were thought to be the result of concerns related to the gender of the baby. In addition, the husband's drinking habits or family clashes may cause tension: "due to tension at home" [staff nurse/ ANM], "repeated birth of female children, leading to mental tension" [ASHA]. Previous caesarean deliveries were also thought to create worry, which could result in high blood pressure. There was some understanding, by health care providers, of underlying conditions that contribute to the hypertensive disorders of pregnancy. These conditions included anaemia and diabetes: "due to anaemia she will have swelling and that will lead to BP" [staff nurse/ANM]. Heredity was also believed to be

Table 2 Focus group discussions

\begin{tabular}{llll}
\hline \# & Stakeholder & Participants & Mean age \\
\hline 1 & Auxiliary Nurse Midwives (ANM) $(n=5)$ & 8 & 36 \\
& Staff nurses $(n=3)$ & 41 \\
2 & Auxiliary Nurse Midwives (ANM) $(n=6)$ & 9 & \\
& Staff nurses $(n=3)$ & & 39 \\
3 & Auxiliary Nurse Midwives (ANM) $(n=10)$ & 19 & 30 \\
& Staff nurses ( $n=7)$ & 12 & 32 \\
4 & Auxiliary Nurse Midwives (ANM) $(n=8)$ & & - \\
& Staff nurses ( $n=4)$ & & \\
5 & Accredited Social Health Activists (ASHA) $(n=10)$ & 10 & 33 \\
6 & Accredited Social Health Activists (ASHA) $(n=11)$ & 11 & \\
7 & Accredited Social Health Activists (ASHA) $(n=15)$ & 15 & \\
8 & Accredited Social Health Activists (ASHA) $(n=17)$ & 17 & \\
\hline
\end{tabular}


associated with pre-eclampsia. Diet was explained as a contributor to the onset of pre-eclampsia: diets high in salt, oil and fried foods. Additionally, strenuous work was seen as a factor that contributes to the development of pre-eclampsia.

Similar factors were believed to be implicated in the development of eclampsia, such as tension or weakness. In addition, some health care providers stated that blood pressure was the cause of eclampsia. This explanation showed an understanding of a relationship between the two conditions. Anaemia was sometimes attributed to the incidence of eclampsia. The most common explanation of the origin of eclampsia was the lack of tetanus toxoid vaccination in pregnancy: "If they don't take TT [tetanus toxoid vaccine] when pregnant, they might get fits" [ANM]. Lastly, poor medical adherence was thought to play a role; the prohibitive cost and the belief that medication will result in big babies prevents some women from appropriately following treatment.

\section{Outcomes of hypertension and convulsions in pregnancy} Health care providers acknowledged that further consequences are likely for women suffering hypertension in pregnancy. In fact, most had a good grasp of these potential outcomes. The most serious was the possibility of death for the mother and/or baby.

The outcomes of eclampsia were thought to be associated with active seizures, disease progression, or death. Many mentioned that injuries might occur, such as broken bones, tongue bite, and aspiration: "She may have injury due to fits episode" [ASHA]. These outcomes may be to the mother, baby or both.

\section{Treatment of hypertension in pregnancy}

Staff nurses and ANMs suggested regular blood pressure measurements in pregnancy. If hypertension was detected they advised as follows: rest in a well-lit and ventilated space, decrease salt intake, iron supplementation, and tetanus vaccination. In addition to these recommendations, staff nurses claimed to provide antihypertensive medication and in some cases $\mathrm{MgSO}_{4}$. ASHAs believed there was a need for regular blood pressure measurement and referral for hypertensive women. ASHAs also stressed the importance of medical adherence and the avoidance of home treatment.

Staff nurses and ANMs who reported use of antihypertensive agents, mostly commonly reported using nifidepine, though others were mentioned: atenolol, amlodipine, beta blockers, diazepam, frusemide, and $\mathrm{MgSO}_{4}$. Some cautioned that these medications should be given by a doctor or after consultation; while others claimed they do not provide such medication under any circumstance. ASHAs were unaware of specific antihypertensive medications, though they recognized the need for appropriate treatment by medical officers or ANMs in cases of hypertension.

\section{Treatment of convulsions in pregnancy}

There was minimal knowledge, confidence or expertise regarding the treatment of convulsions in pregnancy. Some staff nurses stated that $\mathrm{MgSO}_{4}$ was the drug of choice for the treatment of eclampsia; however, few had personal experience administering it, and they were unaware of appropriate routes and dosages. Similarly, all ANMs had some knowledge of $\mathrm{MgSO}_{4}$, but had not administered it themselves. $\mathrm{MgSO}_{4}$ was perceived by some to be treatment-appropriate only in higher-level facilities by specialists. Aside from $\mathrm{MgSO}_{4}$, staff nurses and ANMs expressed familiarity with alternative anticonvulsants: diazepam, frusemide, and phenobarbitone. Some mentioned past use of diazepam, but explained that practice has now changed; however, one staff nurse claimed to still treat eclampsia with diazepam: "We give injection calmpose (diazepam) and send" [staff nurse]. ASHAs did not express familiarity with $\mathrm{MgSO}_{4}$; some knew the name but not the indications for use.

\section{Clinical confidence and competence in identifying and treating hypertension and convulsions in pregnancy}

It is important to identify the confidence of ANMs and staff nurses regarding identification and treatment of the HDPS; this was evaluated using a questionnaire targeting their confidence and experience. The first theme of the questionnaire probed health workers regarding preparedness, awareness, knowledge and skills related to pre-eclampsia and eclampsia. Responses were consistent between respondents with the vast majority of responses being "agree" or "strongly agree". One exception was regarding ANM's and staff nurse's ability to assess proteinuria, where some respondents showed low confidence (Fig. 2).

The second theme of the questionnaire focussed on their scope of practice. Respondents claimed to monitor and refer cases of pre-eclampsia. All 12 reported to either 'agree' or 'strongly agree' to the two related statements: "I monitor women for pre-eclampsia" and "I refer women for pre-eclampsia". The most common response regarding medication used for high blood pressure and seizures was $\mathrm{MgSO}_{4}$. ANMs and staff nurses also claimed to be comfortable counselling women and families to receive $\mathrm{MgSO}_{4}$ prior to referral (Table 3).

\section{Discussion}

The study was conducted to identify the level of knowledge held by community health care workers - staff nurses, ANM, ASHA - regarding pre-eclampsia and eclampsia and their routine management of these cases in 


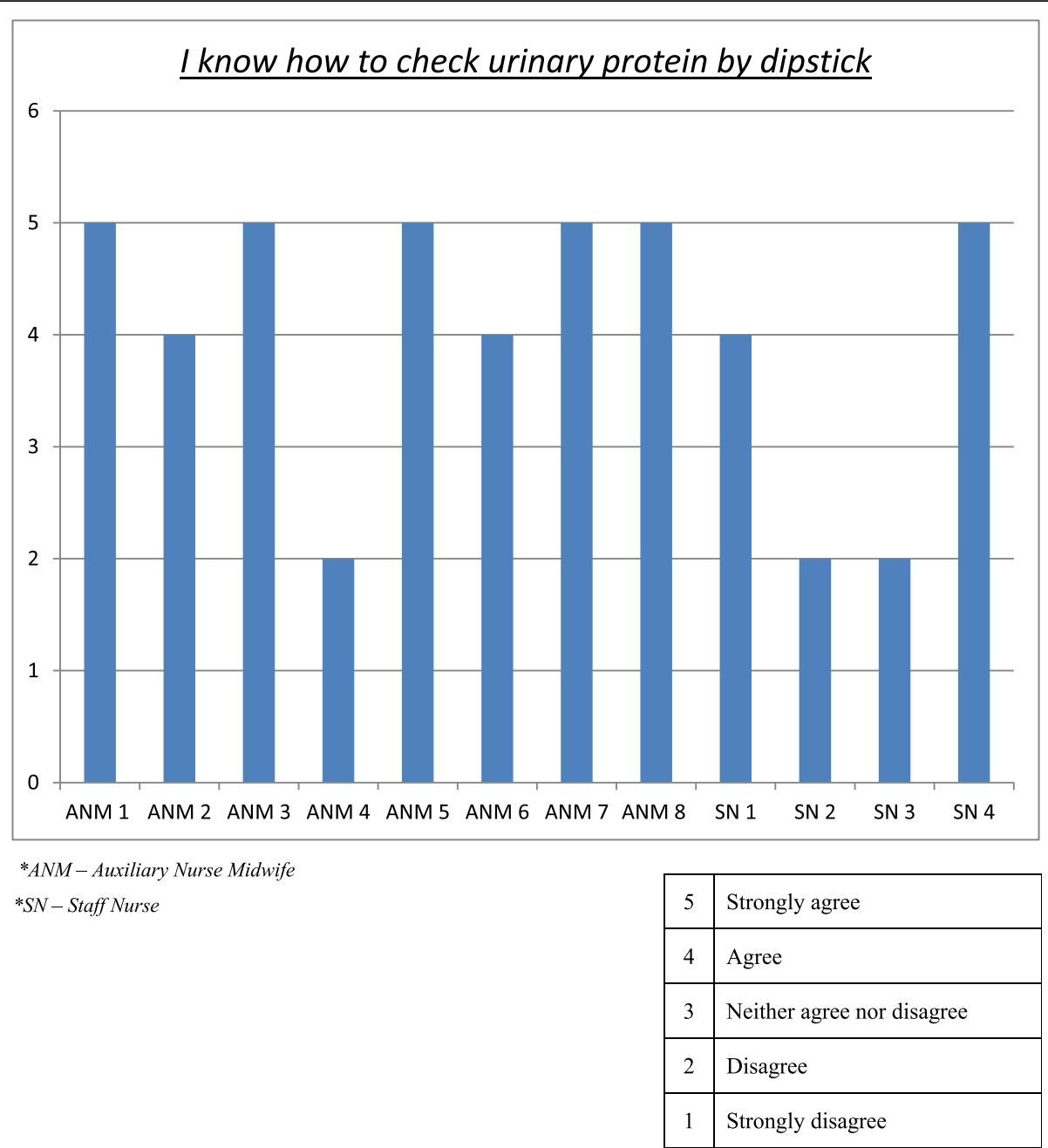

Fig. 2 Ability to measure proteinuria by ANMs and staff nurses

northern Karnataka. Community health workers are essential in identifying obstetric emergencies, thus a sound knowledge of pregnancy complications is needed for early diagnosis and referral. It was observed that most community providers were aware of the link between hypertension and seizures; however, they also mentioned they can present without symptoms.

It was surprising to find that many community health providers believed missing tetanus toxoid vaccinations could lead to eclampsia; this clearly represents a misunderstanding of the underlying cause of disease. Another explanation for the hypertensive disorders of pregnancy was mental tension due to personal conflicts and the preference for a male child. In Indian culture sons are strongly preferred as they are required for the performance of funeral rites, they are expected to provide financially, and in many parts of India they command a large dowry (https://nitawriter.wordpress.com/2006/10/19/whydo-indians-prefer-sons-2/) [14]. The belief that anaemia causes hypertension was stressed by many participants; this misconception may be because of the high prevalence of anaemia in the region. The stated causes indicate significant gaps in knowledge, thus it is necessary to target improvements in the training of community health workers. Nevertheless, community health workers do seem to recognize the serious nature of pre-eclampsia and the urgent need for management and referral.

Health care providers were probed regarding their use and knowledge of antihypertensive medications, to better understand their current practice. ANMs stated that they administered antihypertensive agents when indicated; by far the most common antihypertensive in use was nifidepine. ASHAs were unaware of specific antihypertensive medications, though they recognized they should be administered by medical officers or ANMs for hypertension. It is important for skilled birth attendants to know about antihypertensive drugs, their indications, contraindications, dosage and limitations for their use. 
Table 3 ANM and staff nurse confidence of pre-eclampsia screening

\begin{tabular}{|c|c|c|c|}
\hline Question & $\begin{array}{l}\text { Average } \\
\text { response }\end{array}$ & $\begin{array}{l}\text { Average response } \\
\text { by ANMs } \\
(\mathrm{N}=8)\end{array}$ & $\begin{array}{l}\text { Average response } \\
\text { by staff nurses } \\
(N=4)\end{array}$ \\
\hline I know how to provide routine antenatal care to women in my catchment area & 4.6 & 4.7 & 4.3 \\
\hline I know the danger signals of pregnancy & 4.5 & 4.7 & 4.3 \\
\hline I know how to identify the warning signs of high blood pressure in pregnancy and postpartum & 4.5 & 4.6 & 4.3 \\
\hline I know how to identify the warning signs of seizures in pregnancy and postpartum & 4.5 & 4.5 & 4.5 \\
\hline I know how to identify the symptoms and signs of labour & 4.6 & 4.7 & 4.5 \\
\hline I know how to check blood pressure & 4.7 & 4.8 & 4.5 \\
\hline I know how to check urinary protein by dipstick & 4.0 & 4.4 & 3.3 \\
\hline I know how to check urinary protein by boiling urine & 4.0 & 4.4 & 3.3 \\
\hline I know how to administer vaccines to mothers and children & 4.6 & 4.8 & 4.3 \\
\hline I know how to administer intramuscular injections to pregnant women & 4.7 & 4.8 & 4.3 \\
\hline I know how to administer oral medications to pregnant women & 4.5 & 4.7 & 4.3 \\
\hline I know how to directly refer pregnant women with complications & 4.5 & 4.6 & 4.5 \\
\hline
\end{tabular}

$5=$ strongly agree; 4 = agree; 3 = neither disagree nor agree; 2 = disagree; 1 = strongly disagree

National guidelines authorize ANMs and nurses to administer $\mathrm{MgSO}_{4}$ to women suffering from eclampsia; however, the majority of ANMs claimed not to have administered $\mathrm{MgSO}_{4}$ themselves. There are differences in the professional qualification, job responsibilities, and practical experience of the staff nurses and ANMs; however, practice guidelines are combined and so do not differentiate these responsibilities creating confusion.

Participants stated that they can administer intramuscular injections and oral medications in pregnancy, this selfreported skill can very well be utilized in the management of HDPs; however, it is not clear weather this competence translates to the use of $\mathrm{MgSO}_{4}$. This lack of experience with $\mathrm{MgSO}_{4}$ clearly indicates a gap in knowledge and the implementation of guidelines. Even though community health workers understood that pre-eclampsia and eclampsia can lead to severe outcomes, their knowledge of treatments was inadequate. The majority of ANMs misclassified $\mathrm{MgSO}_{4}$ as an antihypertensive agent and nifedipine as an anticonvulsant for eclampsia. These misunderstandings could lead to adverse effects; in addition, some providers reported use of diazepam which is known to be dangerous in pregnancy. The misclassification of antihypertensives and anticonvulsants by ANMs highlights knowledge gaps that must be addressed.

The large sample size and mixed methods used for this study strengthens the findings. Due to the qualitative nature of this study, the results are not generalizable to other groups or regions. The findings of this study cannot be deemed representative of all community health workers in India; nevertheless they provide insight into the knowledge gaps and common misconceptions related to the hypertensive disorders of pregnancy. Focus group discussions provided detailed insight into the experience and beliefs of community-based health workers; however, the ideas given by a small number of participants may have influenced others. Some focus groups included many participants which may have decreased the facilitator's ability to engage with the whole group; however, this approach allowed participation from a large group of respondents and many potential points of view.

\section{Implications}

Due to the identified knowledge gaps and common misconceptions there is a need to update training and regular assessment of community health worker competence and confidence regarding the identification and treatment of the hypertensive disorders of pregnancy. The national guidelines that authorize ANMs to administer $\mathrm{MgSO}_{4}$ and antihypertensives need to be broadly disseminated so ANMs are fully aware of what their scope of practice includes. Professional practice guidelines that are combined for ANMs and staff nurses should be separated to avoid confusion in their scope of practice. These changes are required at the regulatory and systems level so that ANMs and staff nurses have full clarity and support in provision of appropriate and safe patient care during pregnancy and childbirth.

\section{Conclusion}

Community health care workers are the link between the health care system and the community. The knowledge and practices of these providers can have a profound effect on maternal health outcomes. This study demonstrated that community health workers had limited knowledge of aetiology of pre-eclampsia, as hypertension was most commonly attributed to stress, and 
eclampsia was attributed to a lack of tetanus vaccinations, anaemia, and poor medical adherence. More importantly, most of the health workers acknowledged the serious nature of the disease and were aware of appropriate medications, though not the route and dosage. It is important to build upon the knowledge gaps identified to most effectively improve maternal and perinatal health outcomes.

\section{Additional file}

Additional file 1: Reviewer reports. (PDF 214 kb)

\section{Abbreviations}

ANM: Auxiliary nurse midwife; ASHA: Accredited social health activist; HDP: Hypertensive disorders of pregnancy; LMIC: Low- and middle-income countries; MDG: Millennium development goals; $\mathrm{MgSO}_{4}$ : Magnesium sulphate; MMR: Maternal mortality ratio; NRHM: National Rural Health Mission; PHC: Primary health centre

\section{Acknowledgments}

This work is part of the University of British Columbia PRE-EMPT (Pre-eclampsia/ Eclampsia, Monitoring, Prevention and Treatment) initiative supported by the Bill \& Melinda Gates Foundation. We gratefully acknowledge contributions of the Community Level Interventions for Pre-eclampsia (CLIP) India Feasibility Working Group: Beth Payne, Sharla Drebit, Chirag Kariya, Zulfiqar Bhutta, Anjali Joshi, Laura Magee, Sheela Naik, Sangmesh Rakkareddi, and Amit Revankar. We also acknowledge the support of SN Medical College, KLE University's JN Medical College, University of British Columbia, Government of Karnataka, the district health administration of Belgaum and Bagalkot Districts, the local medical officers, auxiliary nurse midwives, accredited social health activists, and other health centre staff for their participation. We additionally acknowledge the research office staff for assisting in data translation and transcription. Finally, a special thanks to all the participants.

\section{Declarations}

This article has been published as part of Reproductive Health Volume 13 Supplement 2, 2016: Building community-level resilience for the case of women with pre-eclampsia. The full contents of the supplement are available online at http://reproductive-health-journal.biomedcentral.com/articles/supplements/volume-13-supplement-2. Publication charges for this supplement were funded by the University of British Columbia PRE-EMPT (Pre-eclampsia/ Eclampsia, Monitoring, Prevention and Treatment) initiative supported by the Bill \& Melinda Gates Foundation.

\section{Authors' contributions}

UR drafted the manuscript with valuable assistance and input from all authors. MV led analysis of this study. UR, UC, GK, SB, and CK co-ordinated conduct of all study activities. DS and RQ were instrument in the initial study design. AM, SG, PVD and RD provided guidance and oversight during study design, data collection, analysis and interpretation of findings. All authors read and approved the final manuscript.

\section{Competing interests}

The authors declare that they have no competing interests.

\section{Peer review}

Reviewer reports for this article are included in Additional file 1.

\section{Author details}

'Department of Community Medicine, S Nijalingappa Medical College, Bagalkot, Navanagar 587102, India. ${ }^{2}$ Department of Obstetrics and Gynaecology, and the Child and Family Research Unit, University of British Columbia, Vancouver V5Z 4H4, Canada. ${ }^{3}$ KLE University's Jawaharlal Nehru Medical College, Neharu Nagar, Belgaum 590010, India. ${ }^{4}$ Department of Obstetrics and Gynaecology, S Nijalingappa Medical College, Bagalkot, Navanagar 587102, India. ${ }^{5}$ Department of Pharmacology, S Nijalingappa
Medical College, Bagalkot, Navanagar 587102, India. ${ }^{6}$ Department of Research, Vancouver Island Health Authority, Victoria V8R 1J8, Canada. ${ }^{7}$ Division of Women and Child Health, Aga Khan University, Karachi, Pakistan. ${ }^{8}$ Molecularand Clinical Sciences Research Institute, St George's, University of London, and Department of Obstetrics and Gynaecology, St George's University Hospitals NHS Foundation Trust, London SW17 ORE, UK.

${ }^{9}$ Department of Obstetrics and Gynaecology, Thomas Jefferson University, Philadelphia 19107, USA.

Published: 30 September 2016

\section{References}

1. Registrar General of India, Sample Registration System (RGI-SRS) http://pib.nic.in/newsite/mbErel.aspx?relid=124244 visited on 22 Feb 2016

2. SRS Bulletin, Sample Registration System, Registrar, General of India, Ministry of Health, Government of India. 2014; Volume 49 (1)

3. Khan KS, Daniel W, Lale S, Metin Gulmezoglu A. A Van Look PFA: WHO analysis of cause of maternal death: A systematic review. Lancet. 2006; 367(suppl9516):1066-74.

4. Duley L. Maternal mortality associated with hypertensive disorders of pregnancy in Africa, Asia, Latin America and the Caribbean. Br J Obstet Gynaecol. 1992;99:547-53.

5. Ameh CA, Ekechi Cl, Tukur J. Monitoring severe pre-eclampsia and eclampsia treatment in resource poor countries: Skilled birth attendant perception of a new treatment and monitoring chart (LIVKAN chart). Matern Child Health J. 2012;16(5):941-6.

6. Duley L. The global impact of pre-eclampsia and eclampsia. Semin Perinatol. 2009;33(3):130-7. Praveen Kumar, Jai Bhagwan Sharma Hypertensive Disorders in Pregnancy. JIMSA October - December 2010 Vol. 23 No. 4.

7. Sample Registration system: maternal mortality in India: 1997-2003. Trends causes and risk factors. New Delhi Registar General India; 2006. http://www. health.mp.gov.in/Maternal_Mortality_in_India_1997-2003.pdf.

8. Directorate General of Health Services: Indian Public Health Standards(IPHS) for Primary Health Centre revised guidelines. In: Ministry of Health and Family Welfare. New Delhi Government of India; 2010. http://health.bih.nic. in/Docs/Guidelines/Guidelines-PHC-2012.pdf.

9. Directorate General of Health Services: Indian Public Health Standards(IPHS) for Sub Centre revised guidelines. In: Ministry of Health and Family Welfare. New Delhi Government of India; 2010. http://nrhm.gov.in/images/pdf/ guidelines/iphs/iphs-revised-guidlines-2012/sub-centers.pdf.

10. National Rural Health Mission Frame work for implementation (http://nrhm. gov.in/images/pdf/about-nrhm/nrhm-framework-implementation/nrhmframework-latest.pdf).

11. Park K. Park's Text book of Preventive and Social Medicine. 23rd ed. Jabalpur: Banarasidas Bhanot Publishers; 2015.

12. Kishore J. National health programmes of India: National policies and legislations related to health. Peer-reviewed, Official Publication of the Indian Academy of Geriatrics. 2012:165.

13. Khowaja AR, Quershi R, Sawchuck D, Oladapo OT, Adetoro OO, Orenuga EA, Bellad M, Mallapur A, Charanthimath U, Sevene E, Munguambe K, Boene H, Vidler M, Bhutta Z, von Dadelszen P, for the CLIP Working Group. The feasibility of community level interventions for pre-eclampsia in south Asia and sub-Saharan Africa: a mixed-methods design. Reprod Health. 2016;13(Supplement 1):56.

14. Christian D, Sonaliya KN, Garsondiya J. Female feticide in the view of fertile females-A study among suburban pregnant women of Gujarat, India. Int J Med Sci Public Health. 2014;3(3):300-4. 